\title{
Ten-day bismuth-containing quadruple therapy versus 7-day proton pump inhibitor-clarithromycin containing triple therapy as first-line empirical therapy for the Helicobacter pylori infection in Korea: a randomized open-label trial
}

Young-II Kim', Jong Yeul Lee ${ }^{1}$, Chan Gyoo Kim¹, Boram Park², Jin Young Park ${ }^{3}$ and II Ju Choi ${ }^{*}$

\begin{abstract}
Background: This randomized, open-label trial aimed to compare the efficacy of 10-day bismuth-containing quadruple therapy (BQT) with 7-day proton-pump inhibitor-clarithromycin containing standard triple therapy (STT) as an empirical first-line Helicobacter pylori therapy.

Methods: Participants with H. pylori infection were randomly assigned to either 10-day BQT (daily doses of bismuth 300 mg, four times; lansoprazole 30 mg, twice; metronidazole 500 mg, three times; and tetracycline 500 mg, four times) or 7-day STT (lansoprazole 30 mg; amoxicillin 1,000 mg; and clarithromycin 500 mg; each given twice daily). Participants who failed initial therapy were crossed over to the alternative treatment regimen. Primary outcome was the eradication rates of first-line treatment by intention-to-treat analysis.

Results: Study participants $(n=352)$ were randomized to receive either 10-day BQT $(n=175)$ or 7-day STT $(n=177)$. The BQT-group achieved a significantly higher eradication rate than the STT-group in the intention-to-treat analysis (74.3\% vs 57.1\%, respectively; $P=0.001)$, modified intention-to-analysis (87.2\% [130/149] vs 68.7\% [101/147], respectively; $P<0.001)$ and per-protocol analysis $(92.9 \%[105 / 113]$ vs $70.1 \%$ [94/134], respectively; $P<0.001)$. Although there was no serious adverse event, the compliance was lower with BQT than STT as a higher proportion of participants in the BQT-group discontinued therapy because of adverse events than those in the STT-group $(23.1 \%$ vs $9.1 \%$, respectively; $P=0.001)$
\end{abstract}

\footnotetext{
*Correspondence: cij1224@ncc.re.kr

${ }^{1}$ Center for Gastric Cancer, National Cancer Center, 323 Ilsan-ro,

Ilsandong-gu, Goyang, Gyeonggi 10408, South Korea

Full list of author information is available at the end of the article

Disclaimer: Where authors are identified as personnel of the International

Agency for Research on Cancer/World Health Organization, the authors

alone are responsible for the views expressed in this article and they

do not necessarily represent the decisions, policy or views of the

International Agency for Research on Cancer/World Health Organization.
}

(c) The Author(s) 2021. Open Access This article is licensed under a Creative Commons Attribution 4.0 International License, which permits use, sharing, adaptation, distribution and reproduction in any medium or format, as long as you give appropriate credit to the original author(s) and the source, provide a link to the Creative Commons licence, and indicate if changes were made. The images or other third party material in this article are included in the article's Creative Commons licence, unless indicated otherwise in a credit line to the material. If material is not included in the article's Creative Commons licence and your intended use is not permitted by statutory regulation or exceeds the permitted use, you will need to obtain permission directly from the copyright holder. To view a copy of this licence, visit http://creativecommons.org/licenses/by/4.0/. The Creative Commons Public Domain Dedication waiver (http://creativeco mmons.org/publicdomain/zero/1.0/) applies to the data made available in this article, unless otherwise stated in a credit line to the data. 
Conclusions: Ten-day BQT had higher eradication rates compared to that of the 7-day STT as an empirical first-line treatment for H. pylori eradication in Korea.

Trial registration: ClinicalTrials.gov, NCT02557932. Registered 23 September 2015, https://clinicaltrials.gov/ct2/show/ NCT02557932?term $=$ NCT02557932\&draw $=2 \&$ rank $=1$.

Keywords: Helicobacter pylori, Quadruple therapy, Triple therapy

\section{Background}

In the European and North American guidelines on treatment of Helicobacter pylori, the proton pump inhibitor-clarithromycin containing standard triple therapy (STT) without antibiotic susceptibility testing is not recommended as the first-line therapy for the eradication of Helicobacter pylori in areas of high (>15\%) clarithromycin resistance [1, 2]. In Korea, STT has been recommended as the first-line therapy since the $H$. pylori management guidelines were first reported in 1998 [3-5]. A study using a nationwide online registry of $H$. pylori eradication reported that STT was the most common first-line therapy prescribed (91.8\%) in Korea from 2010 to 2015 [6]. However, because of the increased clarithromycin resistance (17.8\%-45.9\%) [7, 8], two meta-analyses including the studies reported from 1998 to 2013 and a nationwide multicenter randomized study reported in 2019 showed that the eradication rates of STT have decreased to unacceptable levels (63.9-74.6\%) in Korea [9-11].

The revised 2013 Korean guideline recommended bismuth-containing quadruple therapy (BQT) as an alternative first-line therapy when clarithromycin resistance is suspected [5]. The European and North American guidelines also recommended the BQT as an empiric first-line therapy in high clarithromycin resistant regions $[1,2]$. However, in the nationwide registry database, the prescription rate of $\mathrm{BQT}$ as first-line therapy in Korea was low (2.2\%) [6]. The low prescription rate of the regimen as first-line therapy is related to the fact that the regimen is reserved for a second-line therapy after the first-line STT failure [5]. The BQT accounts for up to $81 \%$ of all prescribed second-line therapies in Korea [6]. It also has a complex dosing schedule and higher risk of adverse events, which might affect compliance with the regimen [12, 13]. Studies that have evaluated the efficacy of BQT in comparison with the STT have been rarely performed in Korea.

Thus, we conducted a randomized trial to investigate whether 10-day BQT is more effective than the 7-day STT as an empirical first-line therapy for $H$. pylori infection.

\section{Methods \\ Study design}

This study was a single center, open-label, and randomized trial that was conducted at the National Cancer Center, Korea. The institutional review board at the National Cancer Center approved the trial protocol (approval number: NCC2015-0207), and written informed consent was obtained from all participants before enrollment. The trial was registered with ClinicalTrials.gov (number: NCT02557932).

\section{Participants}

Adult participants (aged $\geq 18$ years) were eligible if they had confirmed H. pylori infection and one of following conditions: family history of gastric cancer, postendoscopic resection state for early gastric cancer or adenoma, peptic ulcer disease, or chronic gastritis with non-ulcer dyspepsia. The exclusion criteria included the following: previous H. pylori therapy, history of gastrectomy, cancer of another organ in the last 5 years before enrollment, serious concomitant illnesses, contraindication or allergy history of trial medications, and pregnancy.

The $H$. pylori infection status of the participants at enrollment was evaluated with urea breath test, histological examination by Wright-Giemsa staining of biopsy specimens, and/or rapid urease test. Positive $H$. pylori infection status was confirmed as positivity of any of the following conditions: (1) urea breath test, (2) histological test of at least two biopsy sites, or (3) histological test of one biopsy site with rapid urease test.

\section{Randomization, treatment, and follow-up}

Eligible participants were randomly assigned in a 1:1 ratio to receive either $\mathrm{BQT}$ for 10 days (lansoprazole $30 \mathrm{mg}$, twice daily; tripotassium bismuth dicitrate $300 \mathrm{mg}$, four times daily; tetracycline $500 \mathrm{mg}$, four times daily; and metronidazole $500 \mathrm{mg}$, three times daily) or STT for 7 days (lansoprazole $30 \mathrm{mg}$; clarithromycin $500 \mathrm{mg}$; and amoxicillin 1,000 mg; all given twice daily). A web-based system using computer-generated permuted random block with a size of 2, 4, or 6 was used for randomization, and the stratification factor was sex. The 
randomization sequence was strictly concealed from the study investigators.

The success of $H$. pylori therapy was evaluated at least 6 weeks after completion of the therapy, and the urea breath test was used to determine the $H$. pylori status of the participants. Participants who did not achieve $H$. pylori eradication by the initially assigned treatment regimen, received the crossover therapy; 7-day STT for those who failed the 10-day BQT, and 10-day BQT for those who failed the 7-day STT. Another urea breath test was performed at least 6 weeks after completion of the crossover treatment.

\section{Outcomes}

The primary study outcome was the $H$. pylori eradication rate, as assessed by the urea breath test after completion of the first-line therapy in the intention-to-treat (ITT) population. Secondary outcomes included the following: (1) H. pylori eradication rate of the first-line therapy in the modified ITT population and per-protocol (PP) population, (2) overall $H$. pylori eradication rate after the crossover therapy in the modified ITT population, and (3) adverse events and compliance. All adverse events were recorded according to the common terminology criteria for adverse events (CTCAE) version 4.0, and the drug compliance was evaluated by counting the returned pills of the study drugs. Serious adverse events were assessed for 30 days after the start of the assigned study therapies. The definitions of adverse event severity and serious adverse events are described in Additional file 1: Supplementrary Table 1 and 2, respectively. Compliance was considered low if the participant had consumed less than $80 \%$ of the assigned study drugs.

\section{Statistical analysis}

For sample size calculation, we assumed that the $H$. pylori eradication rates were $74 \%$ for the 7 -day STT and $86 \%$ for the 10 -day BQT. The eradication rate of the 7 -day STT was based on the following assumptions: (1) the clarithromycin resistance rate was $20 \%$, (2) the eradication rates were $95 \%$ for the clarithromycin-susceptible participants and $15 \%$ for the clarithromycin-resistant participants, and (3) 7\% of the participants of follow-up loss. The eradication rate for the 10-day BQT was based on the assumptions of (1) 7\% loss of follow-up participants and (2) 93\% eradication rate of the remaining 93\% participants based on eradication rates reported by previous studies $[14,15]$. With a power of $80 \%$ and a two-sided type 1 error of 5\%, 175 participants were needed for each treatment arm to detect the $12 \%$ difference of eradication rates between two groups ( $74 \%$ vs. $86 \%$ ). An interim analysis was not planned.
The ITT population included all participants who underwent randomization. Participants who did not perform a follow-up urea breath test were considered as eradication failures in the ITT analysis. We performed the modified ITT analysis as an ad hoc analysis, and of the participants who were included in the ITT population, those who did not start the assigned study drugs and those who did not undergo the follow-up urea breath test were excluded from the modified ITT analysis. The PP population included the participants who took at least $80 \%$ of the assigned study drugs and had a followup urea breath test result. Eradication rates were assessed with proportion of successful eradication and $95 \%$ confidence intervals. The safety analysis was performed for the participants who took at least one dose of the assigned treatment. We used the chi-square test or Fisher's exact test to analyze the categorical data, and Student's $t$ test to analyze the continuous data. A two-sided $P$ value of $<0.05$ was considered statistically significant. All statistical analyses were performed using STATA version 16.0 (StataCorp, Texas, USA).

\section{Results}

\section{Study participants}

A total of 574 participants were assessed for eligibility from September 2015 to May 2017. Of these, 352 H. pylori positive participants were randomly assigned to receive either 7-day STT $(n=177)$ or 10 -day BQT $(n=175)$ (Fig. 1). Baseline characteristics were well-balanced between the groups except that the current or former alcohol drinkers were more common in the 10-day BQT group than the 7-day STT group (Table 1).

\section{Eradication rates of first-line therapy}

In the ITT analysis, the eradication rate with the firstline therapy was significantly higher in the participants treated with the 10-day BQT $(74.3 \%, 130 / 175$ participants) than in those treated with the 7-day STT $(57.1 \%$, $101 / 177$ participants; $P=0.001$ ) (Table 2). The post hoc modified ITT analysis also showed significantly higher rate of eradication in the BQT group $(87.2 \%, 130 / 149$ participants) than in the STT group $(68.7 \%, 101 / 147$ participants; $P<0.001)$. In the PP analysis, the BQT group (92.9\%, 105/113 participants) had a higher eradication rate compared with the STT group (70.1\%, 94/134 participants; $P<0.001$ ).

\section{Overall eradication rates after the crossover therapy}

In the modified ITT population for the overall eradication rate, 134 participants from the STT group and 143 from the BQT group were included. In the 7-day STT group, 101 participants who achieved success of $H$. pylori eradication with the first-line and 33 


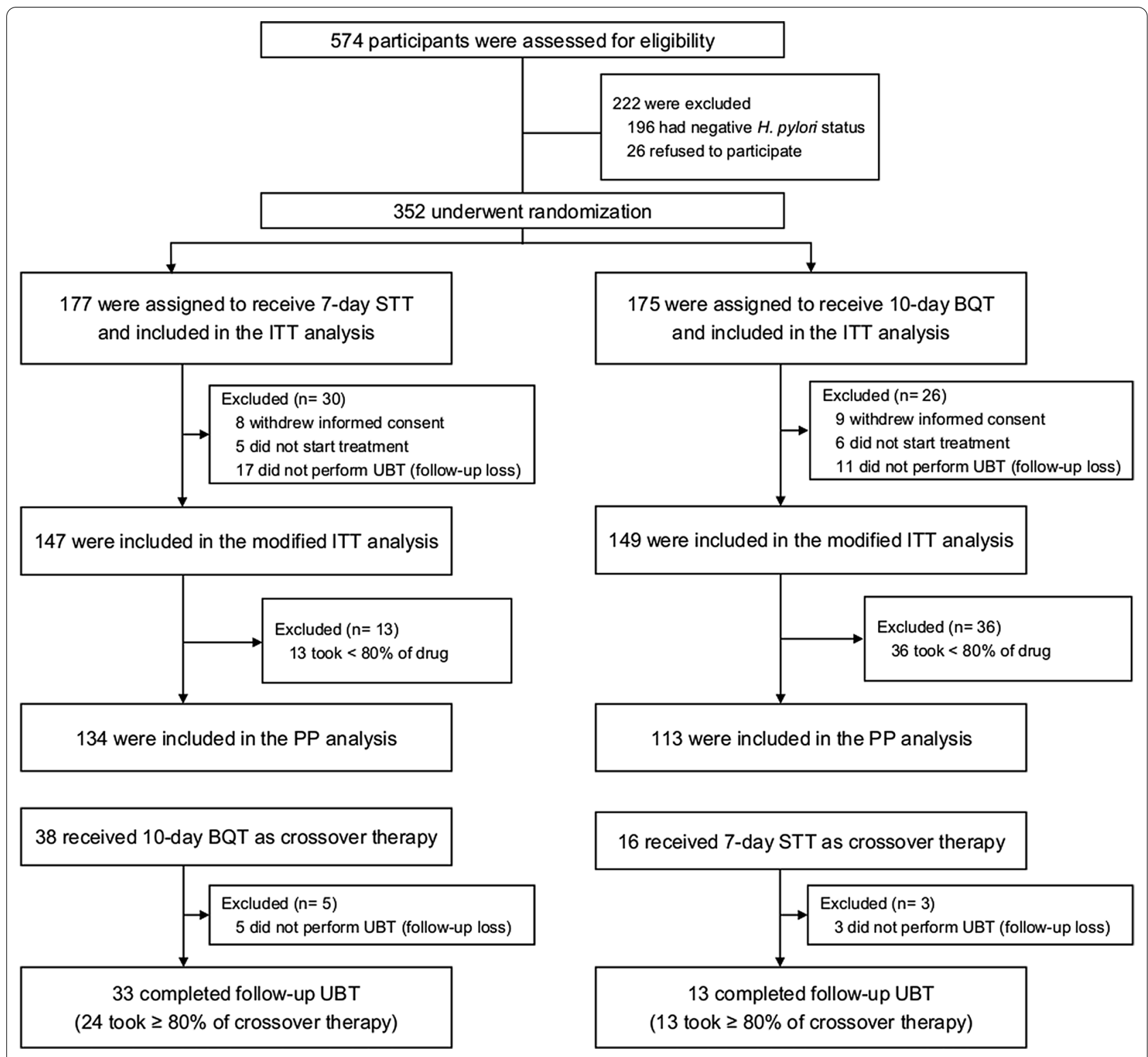

Fig. 1 Study flows. STT, proton-pump inhibitor-clarithromycin containing standard triple therapy; BQT, bismuth-containing quadruple therapy; ITT, intention-to-treat; UBT, urea breath test; PP, per-protocol

participants who started the crossover therapy and underwent follow-up with the urea breath test after the therapy were included. In the 10-day BQT group, $130 \mathrm{H}$. pylori-eradicated participants with the firstline therapy and 13 started the crossover therapy were included. The eradication rate of the 10-day BQT as a crossover therapy was $90.9 \%$ (30/33 participants) and that of the 7-day STT was $84.6 \%$ (11/13 participants). Overall eradication rates were not significantly different between the groups (97.8\% [131/134 participants] for the STT group and 98.6\% [141/143 participants] for the BQT group; $P=0.676$; Table 2). In the ITT and PP analyses, the overall eradication rates were also not different between the groups.

\section{Adverse events and compliance}

In the safety population for the first-line therapy, 164 participants in the 7-day STT and 160 in the 10-day BQT who underwent randomization and received at least one dose allocated treatment were included. The proportion of any adverse events was not significantly different between the treatment groups $(57.3 \%$ in the STT group vs. $67.5 \%$ in the BQT group; $P=0.059$ ) (Table 3 ). However, the incidence of nausea, vomiting, headache, 
Table 1 Baseline characteristics of all participants

\begin{tabular}{|c|c|c|c|}
\hline & $\begin{array}{l}\text { 7-day STT } \\
(n=177)\end{array}$ & $\begin{array}{l}\text { 10-day BQT } \\
(n=175)\end{array}$ & $P$ value \\
\hline Age (year), Mean \pm SD & $55.6 \pm 11.3$ & $53.9 \pm 10.7$ & 0.147 \\
\hline Sex, no. (\%) & & & 0.920 \\
\hline Male & $83(46.9)$ & $83(47.4)$ & \\
\hline Female & $94(53.1)$ & $92(52.6)$ & \\
\hline Smoking, no. (\%) & & & 0.196 \\
\hline Never & $116(65.5)$ & $103(58.9)$ & \\
\hline Current or former smoker & $61(34.5)$ & $72(41.1)$ & \\
\hline Alcohol, no. (\%) & & & 0.028 \\
\hline Never & $82(46.3)$ & $61(34.9)$ & \\
\hline Current or former alcohol drinker & $95(53.7)$ & $114(65.1)$ & \\
\hline Family history of gastric cancer, no. (\%) & $65(36.7)$ & $61(34.9)$ & 0.715 \\
\hline \multicolumn{4}{|l|}{ Coexisting illness, no. (\%) } \\
\hline Hypertension & $37(20.9)$ & $43(24.6)$ & 0.412 \\
\hline Diabetes mellitus & $11(6.2)$ & $13(7.4)$ & 0.651 \\
\hline \multicolumn{4}{|l|}{ Indications for H. pylori treatment, no. (\%) } \\
\hline Post-ESD for adenoma or gastric cancer & $55(31.1)$ & $51(29.1)$ & 0.693 \\
\hline Peptic ulcer disease & $22(12.4)$ & $27(15.4)$ & 0.416 \\
\hline Chronic gastritis with non-ulcer dyspepsia & $100(56.5)$ & $97(55.4)$ & 0.840 \\
\hline
\end{tabular}

STT, proton-pump inhibitor-clarithromycin containing standard triple therapy; BQT, bismuth-containing quadruple therapy; SD, standard deviation; ESD, endoscopic submucosal dissection

Table 2 First-line, crossover and overall eradication rates

\begin{tabular}{|c|c|c|c|c|c|}
\hline & \multicolumn{2}{|l|}{ 7-day STT } & \multicolumn{2}{|l|}{ 10-day BQT } & \multirow[t]{2}{*}{$P$ value } \\
\hline & no./total no. (\%) & $95 \% \mathrm{Cl}$ & no./total no. (\%) & $95 \% \mathrm{Cl}$ & \\
\hline \multicolumn{6}{|l|}{ Eradication rate with first-line therapy } \\
\hline Intention-to-treat analysis ${ }^{\mathrm{a}}$ & 101/177 (57.1) & $49.4-64.5$ & $130 / 175(74.3)$ & $67.1-80.6$ & 0.001 \\
\hline Modified intention-to-treat analysis ${ }^{b}$ & $101 / 147(68.7)$ & $60.5-76.1$ & $130 / 149(87.2)$ & $80.8-92.1$ & $<0.001$ \\
\hline Per-protocol analysis ${ }^{c}$ & $94 / 134(70.1)$ & $61.6-77.7$ & $105 / 113(92.9)$ & $86.5-96.9$ & $<0.001$ \\
\hline \multicolumn{6}{|l|}{ Eradication rate with crossover therapy } \\
\hline Modified intention-to-treat analysis ${ }^{b}$ & 30/33 (90.9) & $75.7-98.1$ & $11 / 13(84.6)$ & $54.6-98.1$ & 0.612 \\
\hline Per-protocol analysis ${ }^{c}$ & $24 / 24(100)$ & $85.8-100$ & $11 / 13(84.6)$ & $54.6-98.1$ & 0.117 \\
\hline \multicolumn{6}{|c|}{ Overall eradication rate after crossover therapy } \\
\hline Intention-to-treat analysis ${ }^{\mathrm{a}}$ & 131/177 (74.0) & $66.9-80.3$ & $141 / 175(80.6)$ & $73.9-86.2$ & 0.142 \\
\hline Modified intention-to-treat analysis ${ }^{b}$ & 131/134 (97.8) & $93.6-99.5$ & 141/143 (98.6) & $95.0-99.8$ & 0.676 \\
\hline Per-protocol analysis ${ }^{c}$ & $117 / 117(100)$ & $96.9-100$ & 108/109 (99.1) & $95.0-100.0$ & 0.482 \\
\hline
\end{tabular}

STT, proton-pump inhibitor-clarithromycin containing standard triple therapy; BQT, bismuth-containing quadruple therapy; $\mathrm{Cl}$, confidence interval

a This analysis was performed in the intention-to-treat population, which included all participants who underwent randomization

b The modified intention-to-treat analysis for the first-line therapy and crossover therapy was performed in participants who started the assigned therapies and underwent the follow-up urea breath test. For overall eradication rate after crossover therapy, participants who achieved eradication success with first-line therapy and those who started the crossover therapy after failure of first-line therapy and underwent the follow-up urea breath test were included

c The per-protocol analysis for the first-line therapy and crossover therapy was performed in participants who took at least $80 \%$ of the therapies and underwent the follow-up urea breath test. For overall eradication rate after crossover therapy, participants who achieved eradication success with first-line therapy in the per-protocol population and those who took at least $80 \%$ of the crossover therapy after failure of first-line therapy and underwent the follow-up urea breath test were included

dyspepsia, general weakness, and anorexia was significantly higher in participants treated with the BQT. Meanwhile, the STT group had a higher proportion of participants with an alteration in taste. All adverse events were grade 1 (mild) or grade 2 (moderate) (Additional file 1: Supplementary Table 3), and none of the 
Table 3 Adverse events and compliance ${ }^{a}$

\begin{tabular}{|c|c|c|c|}
\hline Events & $\begin{array}{l}\text { 7-day STT } \\
(\mathrm{n}=164)\end{array}$ & $\begin{array}{l}\text { 10-day BQT } \\
(n=160)\end{array}$ & $P$ value \\
\hline \multicolumn{4}{|l|}{ Adverse events, no. (\%) } \\
\hline Taste alteration & 47 (28.7) & $7(4.4)$ & $<0.001$ \\
\hline Nausea & $14(8.5)$ & $38(23.8)$ & $<0.001$ \\
\hline Vomiting & $0(0)$ & $10(6.3)$ & 0.001 \\
\hline Diarrhea & $25(15.2)$ & $28(17.5)$ & 0.583 \\
\hline Abdominal discomfort & $25(15.2)$ & $35(21.9)$ & 0.124 \\
\hline Skin rash or urticaria & $2(1.2)$ & $6(3.8)$ & 0.170 \\
\hline Dizziness & $9(5.5)$ & $15(9.4)$ & 0.182 \\
\hline Headache & $5(3.0)$ & $19(11.9)$ & 0.002 \\
\hline Insomnia & $5(3.0)$ & $2(1.3)$ & 0.448 \\
\hline Dyspepsia & $17(10.4)$ & $33(20.6)$ & 0.011 \\
\hline General weakness & $3(1.8)$ & $13(8.1)$ & 0.009 \\
\hline Dyspnea & $1(0.6)$ & $1(0.6)$ & $>0.999$ \\
\hline Anorexia & $0(0)$ & $9(5.6)$ & 0.002 \\
\hline Dry mouth & $5(3.0)$ & $2(1.3)$ & 0.448 \\
\hline Chest discomfort & $0(0)$ & $2(1.3)$ & 0.243 \\
\hline Any adverse event, ${ }^{\mathrm{b}}$ no. (\%) & $94(57.3)$ & $108(67.5)$ & 0.059 \\
\hline Compliance with the study drugs, no. (\%) & & & $<0.001$ \\
\hline Participants who took $\geq 80 \%$ of study drugs & $145(88.4)$ & $117(73.1)$ & \\
\hline Participants who took $<80 \%$ of study drugs & $19(11.6)$ & $43(26.9)$ & \\
\hline Participants with low compliance due to adverse events, ${ }^{\mathrm{C}}$ no. (\%) & $15(9.1)$ & $37(23.1)$ & 0.001 \\
\hline
\end{tabular}

STT, proton-pump inhibitor-clarithromycin containing standard triple therapy; BQT, bismuth-containing quadruple therapy

a This analysis was performed in the safety population, which included all participants who underwent randomization and received at least one dose allocated treatment. Thirteen participants in the 7-day PPI-clarithromycin containing triple therapy group and fifteen in the 10-day bismuth-containing quadruple therapy group were excluded in this analysis

b There was no participant who had any serious adverse events including death, life-threatening events, hospitalization, prolongation of existing hospitalization, persistent or significant disability/incapacity, congenital anomaly/birth defect, and medically important event or reaction

c Low compliance was defined when participant took less than $80 \%$ of the study drugs

participants had serious adverse event. Data on the adverse events related to crossover treatments are shown in Additional file 1: Supplementary Table 4.

Compliance with the study drugs was worse in participants treated with the BQT than in those treated with the STT. The proportion of the participants with low compliance, who took less than $80 \%$ of the assigned study drugs, was $26.9 \%$ (43/160 participants) in those treated with the BQT and $11.6 \%$ (19/164 participants) in participants treated with the STT $(P<0.001$; Table 3$)$. The BQT group had a higher proportion of participants who discontinued the assigned study drugs and consumed less than $80 \%$ of the assigned study drugs because of the adverse events than in the STT group $(23.1 \%$ vs. $9.1 \%$, respectively; $P=0.001$ ).

\section{Eradication rates with the first-line therapy according to drug compliance}

Eradication rates after the initial first-line therapy according to the study drug compliance are presented in Additional file 1: Supplementary Table 5. In the BQT group, the eradication rate of participants who consumed $\geq 80 \%$ of the assigned study drugs $(92.9 \%$, $105 / 113$ participants) was significantly higher than that of participants who consumed $<50 \%$ of the drugs (55.6\%, 10/18 participants; $P<0.001$ ) (Fig. 2). However, despite low compliance $(<80 \%)$ to the study drugs, the eradication rate was $83.3 \%$ (15/18 participants) when participants consumed at least $50 \%$ of the drugs. No significant difference was found in the eradication rate between participants who consumed $\geq 80 \%$ and $50-80 \%$ of the assigned study drugs $(P=0.177)$.

The eradication rate of the participants who completed $50-80 \%$ of the STT $(71.4 \%, 5 / 7$ participants) was not different compared with those who completed $\geq 80 \%$ of that therapy $(70.1 \%, 94 / 134$ participants; $P>0.999)$ (Fig. 2). However, a low eradication rate $(33.3 \%, 2 / 6$ participants) was reported in those who completed less than $50 \%$ of that therapy, although there were no significant differences in eradication 


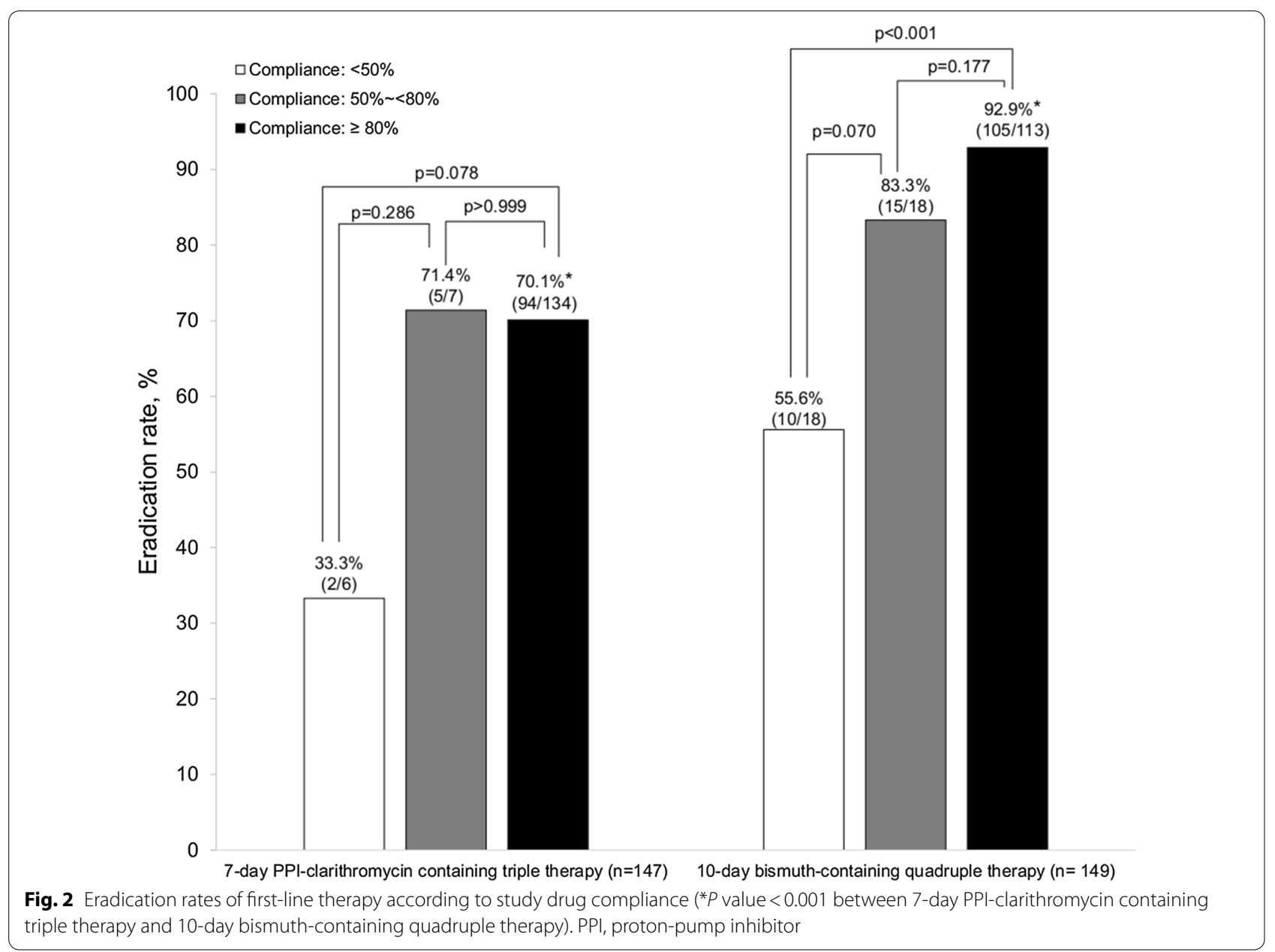

rates compared with those who completed $\geq 80 \%$ or $50 \%-80 \%$ of the therapy.

\section{Discussion}

This randomized trial has shown that the 10-day $\mathrm{BQT}$ is superior to the 7-day STT as an empirical first-line therapy for the H. pylori infection. Although the eradication rate of first-line BQT therapy was low in the ITT population $(74.3 \%)$ because of the poor compliance, that in the PP population was good (92.9\%). Overall combined eradication rates after the crossover therapy were similarly high in both therapy groups.

Our data showed that the eradication rates of the 7-day STT were $57 \%$ in the ITT analysis, $69 \%$ in the modified ITT analysis and 70\% in the PP analysis. The low eradication rates are similar to those reported in a nationwide randomized trial conducted in Korea between 2016 and 2018, which reported the eradication rates of 7-day STT to be $63.9 \%$ in the ITT analysis and $71.4 \%$ in the PP analysis [11]. According to a meta-analysis of nine randomized controlled trials between 2005 and 2013, the overall eradication rate of the STT was $68.5 \%$ in the ITT analysis, and it was inferior to other regimens including the sequential therapy or concomitant therapy as a firstline therapy [9]. In another meta-analysis including 38 randomized controlled trials and 66 observational studies conducted between 1998 and 2013, it was reported that the overall eradication rate of first-line STT was $74.6 \%$ in the ITT analysis, and the rate showed a significantly decreasing tendency from 1998 to 2013 in Korea [10]. The decreasing eradication rates were mainly because of high occurrence of clarithromycin resistance $(>15 \%)$ in Korea $[7,8,16]$. The acceptable targets for the eradication rates of $H$. pylori therapy regimens have been suggested to be $\geq 85 \%$ by the ITT analysis and $\geq 90 \%$ by the PP analysis [17]. Considering these suggestions, the eradication rates of STT reported in our trial and previous studies indicate that STT is unacceptable as a first-line therapy.

In our trial, the crossover 7-day STT in participants who failed to achieve successful eradication in the initial 10-day BQT group showed a higher eradication rate (84.6\%) compared with the first-line 7-day STT (68.7\%). 
The better eradication rate might be associated with a high compliance with the crossover 7-day STT (all participants took $\geq 80 \%$ of provided drugs). In addition, there might be a possibility that most of the 13 participants who received the crossover 7-day STT had clarithromycin-susceptible organism, although clarithromycin resistance was not evaluated.

Our trial showed that the eradication rate of 10-day BQT was $74 \%$ in the ITT analysis, $87 \%$ in the modified ITT analysis and 93\% in the PP analysis, and it was significantly higher than that of the 7-day STT as an empirical first-line therapy. The high eradication rates in our trial were consistent with those reported in previous studies investigating the first-line 10-day BQT in comparison to that of the other regimens $(80-93 \%$ in the intention-totreat analysis and $93-99 \%$ in the per-protocol analysis) $[14,15,18]$. Thus, the 10-day BQT can be an effective empirical first-line therapy alternative to the 7-day STT in Korea.

However, eradication rate of the BQT was $74 \%$ in the ITT analysis, which is unacceptable according to the suggested acceptable target eradication rates of $\geq 85 \%$ by the ITT analysis for the $H$. pylori therapy [17]. In our study, the rate of participants (15.9\%, 56/352 participants) who were lost to follow-up or declined to participate was higher than that we expected (7\%) at sample size calculation. Of 56 participants, 43 (76.8\%) were enrolled for the treatment of H. pylori infection due to chronic gastritis with non-ulcer dyspepsia. Among indications for $H$. pylori treatment of participants who declined to participate in the study or were lost to follow-up, the proportion of chronic gastritis with non-ulcer dyspepsia (21.8\%, 43/197) was a higher than that of post-ESD status $(6.6 \%$, $7 / 106)$ and peptic ulcer disease (12.2\%, 6/49) (Additional file 1: Supplementary Table 6). Because chronic gastritis is not an indication for $H$. pylori eradication in Korea [5], the participants with chronic gastritis might have changed their mind after informed consent and refused to continue participating in the clinical trial. In contrast, the proportion of participants who withdrew consent or lost to follow-up immediately after study enrollment and consequently excluded from the ITT analysis was low in studies conducted outside Korea [14, 15, 18]. Therefore, in our trial, the modified ITT analysis seems to be more reasonable than the ITT analysis in comparing the efficacy with the trials performed in other countries.

Drug compliance with the BQT is important to achieve successful treatment of $H$. pylori. Complex dosing schedule and high frequency of adverse events could reduce compliance for the $\mathrm{BQT}[12,13]$. Regarding the complexity of dosing schedule, previous studies have reported that the modification of drug schedule (twice a day schedule) or use of a single drug containing bismuth, metronidazole, and tetracycline was effective and showed a good compliance $[16,19,20]$. In the present study, low compliance with the BQT seemed to be mainly because of the adverse events, although the frequency of any adverse events was not significantly different between the groups as it was compensated by a higher frequency of taste disturbance observed in the STT group. Most participants treated with the STT could tolerate the adverse events and continued the medication. In a meta-analysis of 41 studies reporting adverse events of the STT, the frequency of adverse events was $20.4 \%$, but the proportion of participants who stopped therapy because of adverse events was low at $1.8 \%$ [10]. Meanwhile, in participants treated with the BQT in the current study, adverse events that affected the drug compliance including nausea, vomiting, headache, dyspepsia, general weakness, and anorexia, were more frequent. Although there was no serious adverse event, BQT group had a higher proportion of participants (23.1\%) discontinued and took less than $80 \%$ of the assigned study drugs because of the adverse events. The eradication rate of the BQT in participants who took less than $80 \%$ of the assigned study drugs $(83 \%$ in those who consumed $50 \%$ to $80 \%$ of the drugs, and $56 \%$ in those who consumed less than $50 \%$ of the drugs) did not reach the acceptable eradication rate of $\geq 85 \%$ [17]. Thus, it is important to provide detailed information to participants on potential adverse events that can occur and emphasize that they should take the prescribed drugs as much as possible if the adverse events are tolerable, to achieve high eradication rate with the BQT. To improve the compliance and reduce adverse events in the 10-day BQT group, additional efforts are needed as follows: (1) use of a single three-in-one capsule containing bismuth, metronidazole, and tetracycline to increase patient convenience [14], (2) modification of drug administration schedule or dosing [13], or (3) adding probiotics [21].

The resistance rates of clarithromycin or metronidazole have been reported to be high $(17.8-45.9 \%$ and $29.5-63.2 \%$, respectively) in Korea [7, 8, 22]. A randomized trial from Taiwan reported that the 10-day BQT was effective as first-line and second-line therapy, with a success rate of $>90 \%$, regardless of the resistance to clarithromycin or metronidazole [18]. Similar to that study results, the BQT in our study was effective and showed a high eradication rate of $91 \%$ as a crossover therapy after failure of the initial STT. Participants who were initially treated with the 7-day STT could achieve an overall eradication rate of $98 \%$ after the crossover therapies, which is the current practice as recommended in the Korean guideline [5]. For those treated by the initial 10-day BQT, overall eradication rate after crossover therapy was also high at $99 \%$. Thus, empirical H. pylori therapy consisting of those two regimens could provide 
final high eradication rates without antibiotic susceptibility testing after the crossover treatment.

In our trial, treatment duration was not the same for the two therapy groups (7 days for STT and 10 days for BQT). International guidelines recommend 14 days for the STT in areas of low clarithromycin resistance $[2,23]$, and 10 or 14 days for the BQT [1, 2, 23-25]. In Korea, 7 to 14 days have been recommended for both regimens [5]. Despite a controversy regarding the optimal duration of STT, meta-analyses reported that extending the duration of the regimen from 7 to 10 or 14 days did not increase the eradication rates significantly in Korea $[9,10]$. In addition, 7 days duration for STT is the most commonly prescribed regimen in Korea ( $84 \%$ of all prescribed first-line regimens) [6]. For the BQT, the eradication rates were higher in the 14 days treatment period as compared to that of the 7 days regimen [26, 27], whereas there was no significant difference between the 14 days and 10 days regimen [25]. Thus, considering the guidelines, drug compliance, and actual clinical practices in Korea, we selected 7 days for the STT and 10 days for the $\mathrm{BQT}$ for comparison in the current study.

As far as we know, this study is the first well-designed prospective randomized controlled trial comparing the 10-day BQT to the 7-day STT as the empirical first-line therapy in Korea. However, there are several limitations to this study. First, we did not evaluate the antibiotic resistance as this study was planned to evaluate the eradication rates in the empirical treatment setting. Second, the proportion of participants who did not return to follow-up visit, including withdrawal from study participation and refusal to receive the assigned study drug, was higher than expected. Thus, we also performed the ad hoc modified ITT analysis after excluding those participants.

\section{Conclusions}

Our trial provides evidence that the 10-day BQT is highly effective in eradicating $H$. pylori as an empirical first-line therapy in Korea. Because the first-line 7-day STT has an unacceptable efficacy, the current Korean guidelines for H. pylori management need to be revised to include BQT as the first-line therapy.

\section{Supplementary Information}

The online version contains supplementary material available at https://doi. org/10.1186/s12876-021-01680-1.

Additional file 1: Supplementary materials

\section{Abbreviations}

STT: Proton-pump inhibitor-clarithromycin containing standard triple therapy; BQT: Bismuth-containing quadruple therapy; ITT: Intention-to-treat; PP: Perprotocol; CTCAE: Common terminology criteria for adverse event.
Acknowledgements

There are no acknowledgements.

\section{Authors' contributions}

IJC designed the research. YIK and BP performed statistical analyses. YIK and IJC wrote the manuscript. YIK, JYP and IJC performed interpretation of data. YIK, JYL, CGK, and IJC enrolled study participants. All authors critically revised the manuscript for important intellectual content. All authors read and approved the final manuscript.

\section{Funding}

This study was supported by a grant from the National Cancer Center, Republic of Korea (Grant number: 1310280, 1311240, 1510530, 1610180, 1810610, 1910370 and 1910270) and partially supported by the grant from the National R\&D Program for Cancer Control, Ministry for Health and Welfare, Republic of Korea (grant number: 20170910). The funders were not involved in the study design, data collection, analysis, data interpretation, and manuscript writing.

\section{Availability of data and materials}

The datasets used and analyzed in the current study are available from the corresponding author on reasonable request.

\section{Ethics approval and consent to participate}

This study was a single center, open-label, and randomized trial that was conducted at the National Cancer Center, Korea. The institutional review board at the National Cancer Center approved the trial protocol (approval number: NCC2015-0207), and written informed consent was obtained from all participants before enrollment.

\section{Consent to publication}

Not applicable.

\section{Competing interests}

The authors declare no conflict of interest.

\section{Author details}

${ }^{1}$ Center for Gastric Cancer, National Cancer Center, 323 IIsan-ro, Ilsandong-gu, Goyang, Gyeonggi 10408, South Korea. ${ }^{2}$ Biostatistics Collaboration Team, Research Core Center, Research Institute, National Cancer Center, Goyang 10408, Korea. ${ }^{3}$ Prevention and Implementation Group, International Agency for Research on Cancer, 69372 Lyon, France.

Received: 22 June 2020 Accepted: 18 February 2021

Published online: 02 March 2021

\section{References}

1. Malfertheiner P, Megraud F, O'Morain CA, Gisbert JP, Kuipers EJ, Axon AT, et al. Management of Helicobacter pylori infection-the Maastricht V/Florence Consensus Report. Gut. 2017;66:6-30.

2. Chey WD, Leontiadis GI, Howden CW, Moss SF. ACG Clinical guideline: treatment of Helicobacter pylori infection. Am J Gastroenterol. 2017;112:212-39.

3. Korean H, Pylori Study Group. Diagnosis and treatment of Helicobacter pylori infection in Korea. Korean J Gastroenterol. 1998;32:275-89.

4. Kim N, Kim JJ, Choe YH, Kim HS, Kim JI, Chung IS. Diagnosis and treatment guidelines for Helicobacter pylori infection in Korea. Korean J Gastroenterol. 2009;54:269-78.

5. Kim SG, Jung HK, Lee HL, Jang JY, Lee H, Kim CG, et al. Guidelines for the diagnosis and treatment of Helicobacter pylori infection in Korea, 2013 revised edition. J Gastroenterol Hepatol. 2014;29:1371-86.

6. Kim BJ, Yang CH, Song HJ, Jeon SW, Kim GH, Kim HS, et al. Online registry for nationwide database of Helicobacter pylori eradication in Korea: correlation of antibiotic use density with eradication success. Helicobacter. 2019;24:e12646.

7. Lee JH, Ahn JY, Choi KD, Jung HY, Kim JM, Baik GH, et al. Nationwide antibiotic resistance mapping of Helicobacter pylori in Korea: a prospective multicenter study. Helicobacter. 2019;24:e12592. 
8. Lee JY, Kim N, Nam RH, In Choi S, Lee JW, Lee DH. Primary and secondary antibiotic resistance of Helicobacter pylori in Korea from 2003 to 2018. Helicobacter. 2019;24:e12660.

9. Lee SW, Kim HJ, Kim JG. Treatment of Helicobacter pylori infection in Korea: a systematic review and meta-analysis. J Korean Med Sci. 2015;30:1001-9.

10. Gong EJ, Yun SC, Jung HY, Lim H, Choi KS, Ahn JY, et al. Meta-analysis of first-line triple therapy for Helicobacter pylori eradication in Korea: is it time to change? J Korean Med Sci. 2014;29:704-13.

11. Kim BJ, Lee H, Lee YC, Jeon SW, Kim GH, Kim HS, et al. Ten-day concomitant, 10-day sequential, and 7-day triple therapy as first-line treatment for Helicobacter pylori infection: a nationwide randomized trial in Korea. Gut Liver. 2019;13:531-40

12. Graham DY, Lee YC, Wu MS. Rational Helicobacter pylori therapy: evidencebased medicine rather than medicine-based evidence. Clin Gastroenterol Hepatol. 2014;12:177-186.e3 (Discussion e12-13).

13. Graham DY, Lee SY. How to effectively use bismuth quadruple therapy: the good, the bad, and the ugly. Gastroenterol Clin North Am. 2015:44:537-63.

14. Malfertheiner P, Bazzoli F, Delchier JC, Celiñski K, Giguère M, Francis M, et al. Helicobacter pylori eradication with a capsule containing bismuth subcitrate potassium, metronidazole, and tetracycline given with omeprazole versus clarithromycin-based triple therapy: a randomised, open-label, non-inferiority, phase 3 trial. Lancet. 2011;377:905-13.

15. Liu KS, Hung IF, Seto WK, Tong T, Hsu AS, Lam FY, et al. Ten day sequential versus 10 day modified bismuth quadruple therapy as empirical firstline and secondline treatment for Helicobacter pylori in Chinese patients: an open label, randomised, crossover trial. Gut. 2014;63:1410-5.

16. Kuo YT, Liou JM, El-Omar EM, Wu JY, Leow AH, Goh KL, et al. Primary antibiotic resistance in Helicobacter pylori in the Asia-Pacific region: a systematic review and meta-analysis. Lancet Gastroenterol Hepatol. 2017;2:707-15

17. Graham DY, Lu H, Yamaoka Y. A report card to grade Helicobacter pylori therapy. Helicobacter. 2007;12:275-8.

18. Liou JM, Fang YJ, Chen CC, Bair MJ, Chang CY, Lee YC, et al. Concomitant, bismuth quadruple, and 14-day triple therapy in the first-line treatment of Helicobacter pylori: a multicentre, open-label, randomised trial. Lancet. 2016;388:2355-65.

19. Kim SJ, Chung JW, Woo HS, Kim SY, Kim JH, Kim YJ, et al. Two-week bismuth-containing quadruple therapy and concomitant therapy are effective first-line treatments for Helicobacter pylori eradication: a prospective open-label randomized trial. World J Gastroenterol. 2019:25:6790-8.

20. Kim JY, Lee SY, Kim JH, Sung IK, Park HS. Efficacy and safety of twice a day, bismuth-containing quadruple therapy using high-dose tetracycline and metronidazole for second-line Helicobacter pylori eradication. Helicobacter. 2020;25:e12683.

21. Wang ZH, Gao QY, Fang JY. Meta-analysis of the efficacy and safety of Lactobacillus-containing and Bifidobacterium-containing probiotic compound preparation in Helicobacter pylori eradication therapy. J Clin Gastroenterol. 2013;47:25-32

22. Savoldi A, Carrara E, Graham DY, Conti M, Tacconelli E. Prevalence of antibiotic resistance in Helicobacter pylori: a systematic review and meta-analysis in World Health Organization regions. Gastroenterology. 2018;155(1372-82):e17

23. Zagari RM, Romano M, Ojetti V, Stockbrugger R, Gullini S, Annibale B, et al. Guidelines for the management of Helicobacter pylori infection in Italy: The III Working Group Consensus Report 2015. Dig Liver Dis. 2015:47:903-12.

24. Liu WZ, Xie Y, Lu H, Cheng H, Zeng ZR, Zhou LY, et al. Fifth Chinese National Consensus Report on the management of Helicobacter pylori infection. Helicobacter. 2018;23:e12475.

25. Fallone CA, Chiba N, van Zanten SV, Fischbach L, Gisbert JP, Hunt RH, et al. The Toronto consensus for the treatment of Helicobacter pylori Infection in adults. Gastroenterology. 2016;151(51-69):e14.

26. Lee BH, Kim N, Hwang TJ, Lee SH, Park YS, Hwang JH, et al. Bismuthcontaining quadruple therapy as second-line treatment for Helicobacter pylori infection: effect of treatment duration and antibiotic resistance on the eradication rate in Korea. Helicobacter. 2010;15:38-45.

27. Chung JW, Lee JH, Jung HY, Yun SC, Oh TH, Choi KD, et al. Second-line Helicobacter pylori eradication: a randomized comparison of 1-week or 2-week bismuth-containing quadruple therapy. Helicobacter. 2011;16:289-94.

\section{Publisher's Note}

Springer Nature remains neutral with regard to jurisdictional claims in published maps and institutional affiliations.
Ready to submit your research? Choose BMC and benefit from:

- fast, convenient online submission

- thorough peer review by experienced researchers in your field

- rapid publication on acceptance

- support for research data, including large and complex data types

- gold Open Access which fosters wider collaboration and increased citations

- maximum visibility for your research: over $100 \mathrm{M}$ website views per year

At BMC, research is always in progress.

Learn more biomedcentral.com/submissions 